\title{
Pneumococcal disease seasonality: incidence, severity and the role of influenza activity
}

\author{
Daniel M. Weinberger ${ }^{1,2,5}$, Zitta B. Harboe ${ }^{3,5}$, Cécile Viboud¹, Tyra G. Krause ${ }^{4}$, \\ Mark Miller ${ }^{1}$, Kåre Mølbak ${ }^{4}$ and Helle B. Konradsen ${ }^{3}$
}

Affiliations: 'Division of International Epidemiology and Population Studies, Fogarty International Center, National Institutes of Health, Bethesda, MD, and ${ }^{2}$ Dept of Epidemiology of Microbial Diseases, Yale School of Public Health, New Haven, CT, USA. ${ }^{3}$ Neisseria and Streptococcus Reference Laboratory, Dept of Microbiology and Infection Control, Statens Serum Institut, Copenhagen, and ${ }^{4}$ Dept of Infectious Disease Epidemiology, Statens Serum Institut, Copenhagen, Denmark. ${ }^{5}$ These authors contributed equally to the work.

Correspondence: Z.B. Harboe, Meningitis and Respiratory Tract Infections, Neisseria and Streptococcus Reference Laboratory, Dept of Microbiology and Infection Control, Division of Diagnostics and Infection Control, Statens Serum Institut, Artillerivej 5, DK-2300 S Copenhagen, Denmark. E-mail: zitassi.dk

ABSTRACT We tested whether the effect of influenza activity on invasive pneumococcal disease incidence and severity varies between age and comorbidity groups.

Weekly rates of invasive pneumococcal disease were obtained from the Danish National Laboratory Surveillance System (1977-2007). Influenza-like illness data were collected from a sentinel surveillance system at the Statens Serum Institut (Copenhagen, Denmark). We fitted Poisson regression models for invasive pneumococcal disease, with predictors of seasonality, trends and influenza activity, and allowed the influenza activity variable to vary by comorbidity level and clinical presentation.

Influenza activity accounted for $8.4 \%$ (95\% CI 4.8-11.9\%) and 6.9\% (95\% CI 5.4-10.2\%) of all invasive pneumococcal disease cases among those aged $15-39$ and $\geqslant 40$ years, respectively, but had no measurable impact among children aged $<15$ years. Influenza activity was associated with significant increases in the incidence of invasive pneumococcal pneumonia in both children and adults. The association was more pronounced among younger adults without comorbidities. Case fatality also varied seasonally among the elderly, and this variation might be associated with influenza activity.

Pneumococcal incidence and the severity of disease varied seasonally and between age groups. The effect of influenza activity on pneumococcal disease varied between children and adults, and this difference was largely due to differences in disease presentation.

@ERSpublications

Pneumococcal incidence and the severity of disease varied seasonally and between age groups http://ow.ly/siBvx

This article has supplementary material available from www.erj.ersjournals.com

Received: March 292013 | Accepted after revision: July 192013 | First published online: Sept 132013

Support statement: This work is conducted in the context of the Multinational Influenza Seasonal Mortality Study (MISMS), with funding from the Office of Global Health Affairs' International Influenza Unit in the Office of the Secretary of the Department of Health and Human Services.

Conflict of interest: Disclosures can be found alongside the online version of this article at www.erj.ersjournals.com

Copyright @ERS 2014 


\section{Introduction}

Streptococcus pneumoniae, or pneumococcus, causes a large proportion of pneumonia cases worldwide [1], as well as meningitis, septicaemia and otitis media. While paediatric conjugate vaccines have successfully reduced disease burden in vaccinated age groups [2], pneumococcal pneumonia remains a significant cause of morbidity, particularly in adults. Understanding the aetiology of pneumococcal disease in different risk groups can help to inform clinical decision making, to determine the potential impact of viral epidemics and pandemics, and to better tailor public health interventions to prevent disease.

Pneumococcal disease frequently occurs following a primary viral infection, and the importance of prior or concomitant infection with influenza virus has been particularly well documented [3-10]. Evidence from epidemiological studies and experimental animal models support a role for influenza in affecting both transmission and host susceptibility to pneumococcus [9, 11-17].

Host characteristics probably affect the synergistic relationship between influenza and pneumococcus. During the 2009 influenza A (H1N1) pandemic, the effect of influenza on pneumococcal pneumonia varied significantly between age groups, with a particularly strong effect among school-aged children [10]. Aside from age, comorbidities could influence the relative importance of influenza. In a study of severe pandemic influenza infections in intensive care units, patients with bacterial co-infections tended to be younger and with fewer underlying conditions than patients without co-infections [18].

In the present study, we used data from Danish national registries spanning three decades to determine whether the importance of the interaction between influenza activity and pneumococcus varies with age and underlying conditions.

\section{Methods}

Data sources

Invasive pneumococcal disease (IPD) data were obtained from the Danish National Laboratory Surveillance System at the Statens Serum Institut (SSI; Copenhagen Denmark), which has maintained individual-level information on pneumococcal disease patients since 1977 [19, 20]. All IPD cases were used for estimation of the seasonal incidence curves, and all cases that occurred during years in which weekly influenza data were available (1982/1983-1990/1991 and 1994/1995-2006/2007) were used to fit the models. Cases of invasive pneumococcal pneumonia (IPP) were defined as the occurrence of culture confirmed pneumococcal bacteraemia in a hospitalised patient who received a discharge code of pneumonia (codes 073, 471, 480-486 from the World Health Organization International Classification of Disease (ICD)-8 and codes J12-J18 from ICD-10 [20]. Date of hospital admission, patient age, clinical presentation (IPD, with or without a pneumonia diagnosis) and comorbidities were compiled for each patient. A summary index for patient comorbidity was estimated by the Charlson index as previously described [20]. Weekly time series were created for each age group $(0-4,5-14,15-24,25-39,40-64$ and $\geqslant 65$ years), clinical presentation and comorbidity group, as indicated. For the regressions, the time series were collapsed into broader age groups to increase statistical power $(0-14,15-39$ and $\geqslant 40$ years $)$.

Weekly influenza-like illness (ILI), defined as acute onset of fever, myalgia and respiratory symptoms, data were used as a proxy for influenza viral activity [21, 22]. The data were collected from two sources, both managed by SSI. The Danish sentinel surveillance system, covering the period 1994/1995-2006/2007, is based on voluntary participation of up to 150 nationwide general practitioners. Between weeks 40 and 20 of the following calendar year, general practitioners report the weekly number of ILI consultations and the number of total consultations in their practice [23]. For 1982/1983-1990/1991, data were obtained from nationwide reports of general practitioners. Weekly ILI data were not available between 1991/1992 and 1993/1994. The ILI data from both surveillance systems were scaled by dividing by the mean winter activity for the respective surveillance system and then subtracting the mean summer value to anchor the minimum values to 0 .

The study was approved by the Danish Data Protection Agency (record number 2007-41-0229).

\section{Seasonality of IPD incidence}

We determined the total number of cases of IPD in each July-June period from 1977/1978-2006/2007 and the percentage of this total occurring in each week. We then calculated the mean percentage and $95 \%$ confidence interval for each week using data from all the years, with the average weighted by the total number of isolates in the July-June period.

\section{Effect of ILI on pneumococcal incidence}

To determine the effect of ILI on the incidence of IPD, we fitted a Poisson regression model based, in part, on methods described elsewhere $[3,8,10]$. Respiratory seasons ran from July to June of each year and, for 
these analyses, we focused only on the seasons with available data on ILI activity ( $\mathrm{n}=22$ seasons, 1982/19831990/1991 and 1994/1995-2006/2007). As indicated, data were stratified by clinical presentation (IPD with or without a pneumonia diagnosis) and Charlson comorbidity score.

Akaike information criteria were used to identify the most parsimonious model. The best model included 52 -week harmonic terms that varied between strata as well as 26-week harmonic terms and dummy variables for weeks 51-52 and 1-3 that did not vary between strata. Alternative models included simpler ones that fitted a single seasonal baseline for all strata or more complicated models that allowed all of the components to vary between strata. The final model was:

$$
\begin{gathered}
\ln \left(\mathrm{Y}_{\mathrm{ij}} / \mathrm{Z}_{\mathrm{j}}\right)=\alpha+\tau_{\mathrm{j}}+\beta_{\mathrm{m}} \times \text { week }_{\mathrm{m}}+\gamma_{\mathrm{q}} \times \sin \theta_{\mathrm{q}}+\delta_{\mathrm{q}} \times \cos \theta_{\mathrm{q}}+\varepsilon \times \text { flu }+\kappa_{\mathrm{j}} \times \text { flu }+ \\
v_{52 \mathrm{j}} \times \sin \theta_{52}+\varphi_{52 \mathrm{j}} \times \cos \theta_{52}
\end{gathered}
$$

where $Y_{i j}$ is the weekly number of pneumococcal cases in week $i$ for comorbidity and syndrome stratum $j$, and $Z_{j}$ is an offset representing the mean number of cases during non-ILI summer weeks (July-November and April-June of each respiratory season). $\beta, \gamma, \varepsilon$ and $\kappa$ are regression coefficients for the respective variables. This offset effectively adjusts for changes in detection/reporting, multi-year changes in incidence and changes in population size. $\alpha$ and $\tau_{j}$ are the overall and strata-specific intercepts. Week $k_{m}$ is an indicator variable for weeks 51,52,1, 2 and 3 of the year, which controls for the mid-winter spike and drop in incidence; $\sin \theta \mathrm{q}$ and $\cos \theta \mathrm{q}$ are harmonic terms representing annual and semi-annual periodicities (52- and 26-week) to control for baseline seasonal fluctuations. Flu represents weekly ILI activity, lagged by 1 week (lags of 0 and 2 weeks were also evaluated and gave similar results). For the "flu" and the 52-week harmonic terms, we estimated both an overall average effect and a stratum-specific effect (interactions with dummy variable for stratum). The combination of $\varepsilon$ and $\kappa_{j}$ gives the overall effect of ILI. The model was fitted separately to data from each age group, and the ILI-attributable fraction of pneumococcal disease was calculated as described elsewhere $[8,10]$. Confidence intervals were calculated using seasonal block bootstraps [24] with 1000 replicates.

In sensitivity analyses, we fitted the model to data from each stratum separately and found the overall patterns to be the same, albeit with more variability. We also fitted a simplified model where the seasonal harmonic terms did not vary between strata, and this did influence the estimates of the "flu" effect, as described. The simplified model was:

$$
\ln \left(\mathrm{Y}_{\mathrm{ij}} / \mathrm{Z}_{\mathrm{j}}\right)=\alpha+\tau_{\mathrm{j}}+\beta_{\mathrm{m}} \times \text { week }_{\mathrm{m}}+\gamma_{\mathrm{q}} \times \sin \theta_{\mathrm{q}}+\delta_{\mathrm{q}} \times \cos \theta_{\mathrm{q}}+\varepsilon \times \text { flu }+\kappa_{\mathrm{j}} \times \text { flu }
$$

\section{Seasonality of case fatality ratio and effect of ILI activity by age group}

To test whether the case fatality ratio of IPP varied seasonally and whether some of these variations could be associated with ILI activity, we used logistic regression models (binomial distribution and log link). The end-point was death due to any cause occurring within 30 days of an IPD-related admission [20]. The model outcome was deaths/IPP cases and predictors included ILI epidemic period (dichotomous) and month of the year, as indicated. For the model that included month of the year, there were indicator variables for each month between November and March, and the reference group was April-September. Serfling regression [25] was used to determine the seasonal baseline for ILI and to define "ILI epidemic" weeks. The risk ratios were calculated by exponentiation of the relevant regression coefficients. The analysis was stratified by age group (40-64 years and $\geqslant 65$ years) and comorbidity level (low Charlson score of 0 , or medium/high Charlson score (score of 1 or more)). Younger age groups had too few deaths for analysis and, due to sparser numbers in the early years, we focused on the period 1994/1995-2006/2007 for this analysis.

Models were fitted using PROC GENMOD, SAS software, version 9.2 (SAS Institute, Cary, NC, USA).

\section{Results}

Population and disease characteristics

From 1977-2007, there were 18858 cases of IPD in Denmark reported to the SSI, of which 11878 (63\%) had an ICD-coded diagnosis of pneumonia. Of these, 47\% $(n=5633)$ had medium or high Charlson comorbidity scores $(\geqslant 1)$ (table 1 ). Most patients aged $<65$ years with IPP had a low level of comorbidity as estimated by the Charlson index (score of 0 ), while the majority of the cases in the $\geqslant 65$ years population had medium/high comorbidity scores (score of $\geqslant 1$ ) (table 1 ).

The incidence of IPD in all age groups demonstrated winter seasonality (fig. 1). Among those aged $\geqslant 65$ years, there was a broad seasonal curve with the minimum incidence occurring in August and a sharp spike in disease that occurred during the last weeks of December, corresponding to the Christmas holiday (fig. 1e). A similar pattern was observed in the 40-64-year-old population, although the late-December spike in incidence was less pronounced in this group (fig. 1d). The 0-4-, 5-14-, 15-24- and 25-39-year-old populations had broad seasonal peaks without a strong winter spike and, in all age groups, there was a 
TABLE 1 Cases of invasive pneumococcal disease (IPD) by age group and Charlson comorbidity score $1977-2007^{\#}$

\begin{tabular}{|c|c|c|}
\hline Age years & Invasive pneumonia & Other IPD \\
\hline \multicolumn{3}{|l|}{$<5$} \\
\hline Total & 394 & 1187 \\
\hline Low comorbidity (Charlson 0) & 337 & 1125 \\
\hline Medium/high comorbidity (Charlson $\geqslant 1$ ) & 57 & 62 \\
\hline \multicolumn{3}{|l|}{ 5-14 } \\
\hline Total & 188 & 224 \\
\hline Low comorbidity (Charlson 0) & 166 & 184 \\
\hline Medium/high comorbidity (Charlson $\geqslant 1$ ) & 22 & 40 \\
\hline \multicolumn{3}{|l|}{$15-24$} \\
\hline Total & 215 & 112 \\
\hline Low comorbidity (Charlson 0) & 186 & 83 \\
\hline Medium/high comorbidity (Charlson $\geqslant 1$ ) & 29 & 29 \\
\hline \multicolumn{3}{|l|}{$25-39$} \\
\hline Total & 1140 & 532 \\
\hline Low comorbidity (Charlson 0) & 955 & 415 \\
\hline Medium/high comorbidity (Charlson $\geqslant 1$ ) & 185 & 117 \\
\hline \multicolumn{3}{|l|}{ 40-64 } \\
\hline Total & 3507 & 2182 \\
\hline Low comorbidity (Charlson 0) & 2065 & 1245 \\
\hline Medium/high comorbidity (Charlson $\geqslant 1$ ) & 1442 & 937 \\
\hline \multicolumn{3}{|l|}{$\geqslant 65$} \\
\hline Total & 6434 & 2743 \\
\hline Low comorbidity (Charlson 0) & 2536 & 1224 \\
\hline Medium/high comorbidity (Charlson $\geqslant 1$ ) & 3898 & 1519 \\
\hline
\end{tabular}

Data are presented as $\mathrm{n} .{ }^{\#}: \mathrm{N}=18858$.

modest drop in incidence during late December/early January (figs $1 \mathrm{a}-\mathrm{c}$ ). In patients aged $\geqslant 65$ years, the seasonal distributions were comparable regardless of comorbidity (online supplementary fig. S1).

The effect of ILI varies by age group and clinical manifestation

We first estimated the association between ILI and the incidence of IPD. ILI was associated with significant increases in the incidence of IPD above the seasonal baseline among 15-39- and $\geqslant 40$-year-olds. In these age groups, $8.4 \%$ (95\% CI $4.8-11.9)$ and $6.9 \%$ (95\% CI 5.4-10.2) of IPD cases were attributable to ILI, respectively. In contrast, there was no detectable increase in the incidence of IPD above the seasonal baseline among the $<15$-year-olds (aetiological percentage $0.6 \%$, 95\% CI $-3.8-5.5 \%$ ).

Most IPD cases in adults presented with pneumonia, while most cases in children did not (table 1). To evaluate whether this difference in disease presentation might explain the lack of a detectable effect in children, we next stratified the cases based on whether they had a clinical diagnosis of pneumonia. Among those aged $<15$ years, ILI was associated with a significant percentage of IPD cases with a pneumonia diagnosis (aetiological fraction 7.7\%, 95\% CI 0.9-14.0\%), but there was no detectable effect of ILI on the incidence of IPD without a pneumonia diagnosis $(-3.1 \%, 95 \%$ CI $-8.9-3.2 \%)$ (fig. 2). The effect of ILI on the incidence of IPP was comparable between children and adults (fig. 2). Among adults, ILI had a significant impact on the incidence of IPD with or without a pneumonia diagnosis (fig. 2).

\section{Comorbidity and the effect of ILI}

We next considered whether underlying conditions might affect the ILI-IPD interaction. For the age groups where we had sufficient data, we stratified the analysis by Charlson comorbidity score. Among the 15-39-yearolds, ILI was associated with a significant increase in IPD among those with low comorbidity levels (aetiological fraction 9.9\%, 95\% CI 6.0-13.0\%) but had no detectable effect among those with known comorbidities (aetiological fraction $0.3 \%, 95 \%$ CI $-8.4-9.7 \%$ ) (table 2). In contrast, among those aged $\geqslant 40$ years, the effect of ILI was of a comparable magnitude among those with or without underlying conditions (table 2). This general pattern was maintained when we further stratified the data based on clinical manifestation (table 2), although the confidence intervals were broad due to small numbers of patients in each stratum. 

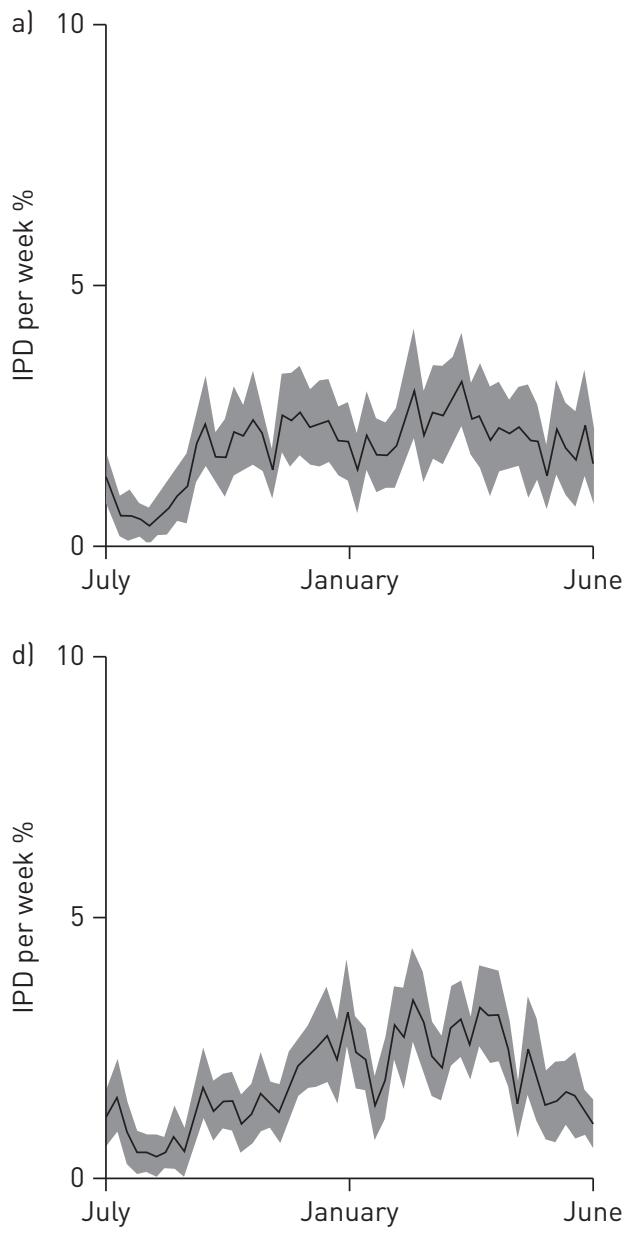

b)

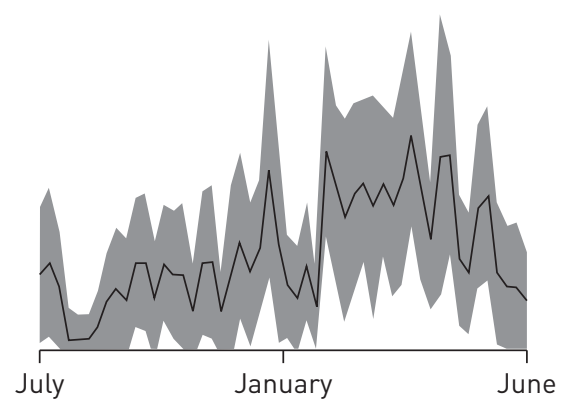

e)

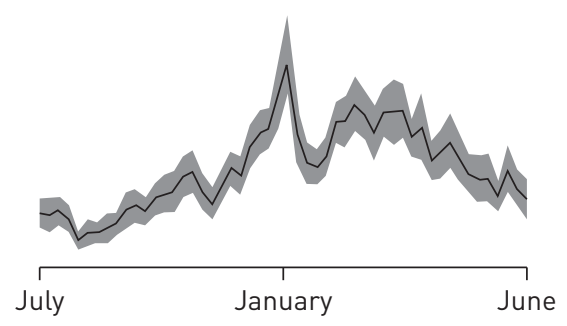

c)

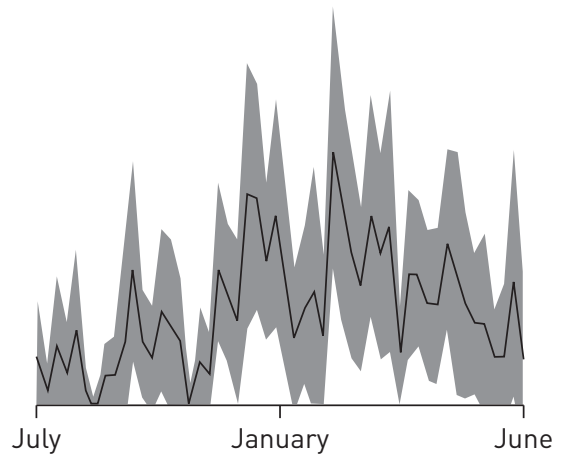

f)

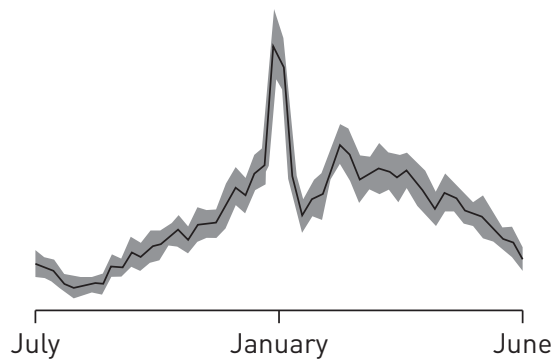

FIGURE 1 Seasonal fluctuations in the incidence of invasive pneumococcal disease (IPD), expressed as percentage of disease occurring in each week out of the total cases in each July-June season for those aged a) $<5$, b) 5-14, c) $15-24$, d) $25-39$, e) $40-64$ and f) $\geqslant 65$ years. Shading shows the $95 \%$ confidence intervals by age group.

\section{Severity of IPP is seasonal}

Finally, we considered whether pneumococcal disease severity, in addition to incidence, varies seasonally and whether ILI might influence these variations. Case fatality ratios for IPP demonstrated significant seasonal variation in the population aged $\geqslant 65$ years. Those with low comorbidity scores had an increased risk of death in January compared with April-October (risk ratio (RR) 1.71, 95\% CI 1.17-2.49) (table 3). The risk of death in this group was elevated for the period from November to March, although only the increase in January was statistically significant. Among the $\geqslant 65$-year-olds with medium/high comorbidity scores, the risk of death was significantly higher in December (RR 1.37, 95\% CI 1.14-1.65) and February (RR 1.31, 95\% CI 1.06-1.62) compared with April to October (table 3). In contrast, among the 40-64-yearolds, there were no significant increases in case-fatality rates during the winter months (table 3 ).

In a multivariate model that included terms for month of the year and ILI activity (ILI activity above a seasonal threshold), we found that the risk of death increased by $42 \%$ during ILI epidemic periods among those aged $\geqslant 65$ years with low comorbidity scores (RR 1.42, 95\% CI 1.06-1.90). ILI activity was not associated with any detectable change in the population-level risk of death due to IPP among the $\geqslant 65$ years population with medium/high comorbidity scores or among 40-64-year-old cases, regardless of comorbidity.

\section{Discussion}

Based on data spanning three decades and a dataset of almost 19000 IPD cases, we demonstrate that the incidence and severity of IPD both vary seasonally, and these seasonal patterns differ between age groups. The relative importance of ILI in driving the incidence of IPD varied between age groups, and some of this variation can be explained by differences in clinical manifestation (pneumonia in older age groups and nonpneumonia in younger age groups). The importance of ILI might also vary by host comorbidity, but the numbers of cases in the stratified analyses were too small to draw firm conclusions. These findings are 


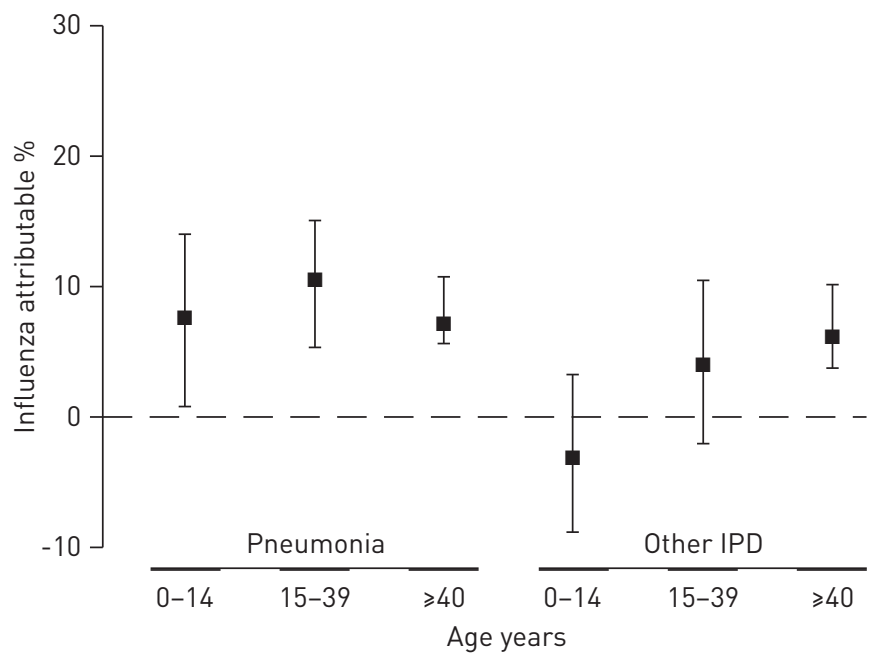

FIGURE 2 Effect of influenza-like illness on incidence of invasive pneumococcal disease (IPD) by age and clinical manifestation (IPD cases with or without a diagnosis of pneumonia). 95\% confidence intervals are shown.

significant because they suggest that the characteristics of patients presenting with pneumococcal disease will differ seasonally and during influenza epidemic/pandemic periods and noninfluenza periods. This information could be used to improve planning for pandemic scenarios.

Our estimates of the fraction of IPD attributable to ILI are largely in line with previous estimates from Sweden (6-7\%) [3] and the USA (4.5-6\%) [8]. In the US study, they found that the effect of influenza was detectable only among pneumonia cases. In contrast, we found that influenza activity was associated with significant increases in the incidence of IPD without a pneumonia diagnosis among adults not among children. It is possible that the pneumonia case definitions used in this study were different from those in the USA, where an active surveillance system is in place. However, the proportion of adult IPD cases with a pneumonia diagnosis is comparable in the two populations.

It is unclear why influenza would be associated with increases in nonpneumonia IPD cases. One possibility is that the observed increases in adult IPD result from both increased susceptibility to pneumococcal invasion in virally infected individuals and increased transmission of the bacteria in the population, which would influence IPD incidence in individuals with or without a primary viral infection. Also, as this is a registerbased study, some confounding could occur when assigning pneumonia diagnosis codes to patients admitted to a hospital with IPD. However, this would not be expected to be systematic throughout the dataset.

The seasonal patterns of IPD incidence among Danish adults mirrored those described in the USA [26, 27], with a sharp mid-winter spike that corresponds to the Christmas holidays. However, we found that the seasonal pattern in younger age groups was distinct; not only was there a lack of a winter spike, but there

TABLE 2 Percentage of invasive pneumococcal disease (IPD) attributable to influenza-like illness activity in Denmark during 1977-2007, stratified by comorbidity and by clinical manifestation

Age years

$15-39 \geqslant 40$

$\begin{array}{lcc}\text { All IPD } & & \\ \quad \text { Low comorbidity (Charlson 0) } & 9.9(6.0-13.0) & 7.6(5.1-11.6) \\ \quad \text { Medium/high comorbidity (Charlson } \geqslant 1) & 0.3(-8.4-9.7) & 6.2(4.3-9.3) \\ \text { IPD with pneumonia diagnosis } & & 7.8(5.8-11.7) \\ \quad \text { Low comorbidity (Charlson 0) } & 11.2(6.5-14.8) & 6.5(4.4-10.1) \\ \quad \text { Medium/high comorbidity (Charlson } \geqslant 1) & 5.4(-5.0-18.7) & 6.9(1.8-12.8) \\ \text { IPD with other diagnosis } & & 5.3(2.5-8.9) \\ \quad \text { Low comorbidity (Charlson 0) } & 6.6(-1.2-14.3) & \end{array}$

Data are presented as \% (95\% CI). * : N=13882 cases of IPD; those aged $<14$ years were excluded from this analysis due to small numbers in the medium/high comorbidity group. 
TABLE 3 Risk of death from invasive pneumococcal pneumonia in each month compared with July-September by age group and Charlson comorbidity score during 1994-2007

\begin{tabular}{|c|c|c|c|c|c|}
\hline Age years & Month & Die $n$ & Survive $\mathrm{n}$ & Case fatality \% & Risk ratio $(95 \% \mathrm{Cl})$ \\
\hline \multicolumn{6}{|l|}{$40-64$} \\
\hline \multirow{3}{*}{ Low comorbidity (Charlson 0) } & January & 16 & 150 & 9.6 & $1.0(0.6-1.7)$ \\
\hline & February & 12 & 166 & 6.7 & $0.7(0.4-1.3)$ \\
\hline & March & 11 & 196 & 5.3 & $0.6(0.3-1.1)$ \\
\hline \multirow{5}{*}{ Medium/high comorbidity (Charlson $\geqslant 1$ ) } & December & 18 & 112 & 13.9 & $0.8(0.5-1.3)$ \\
\hline & January & 21 & 75 & 21.9 & $1.2(0.8-1.9)$ \\
\hline & February & 22 & 102 & 17.7 & $1.0(0.6-1.7)$ \\
\hline & March & 11 & 117 & 8.6 & $0.7(0.4-1.3)$ \\
\hline & April-October & 81 & 381 & 17.5 & Reference \\
\hline \multicolumn{6}{|l|}{$\geqslant 65$} \\
\hline \multirow{2}{*}{ Low comorbidity (Charlson 0) } & March & 30 & 169 & 15.1 & $1.3(0.9-2.0)$ \\
\hline & April-October & 68 & 540 & 11.2 & Reference \\
\hline \multirow[t]{6}{*}{ Medium/high comorbidity (Charlson $\geqslant 1$ ) } & November & 45 & 203 & 18.2 & $0.9(0.7-1.2)$ \\
\hline & December & 129 & 328 & 28.2 & $1.4(1.1-1.6)^{\#}$ \\
\hline & January & 68 & 225 & 23.2 & $1.1(0.9-1.4)$ \\
\hline & February & 84 & 227 & 27.0 & $1.3(1.1-1.6)^{\#}$ \\
\hline & March & 69 & 238 & 22.5 & $1.1(0.9-1.4)$ \\
\hline & April-October & & & & Reference \\
\hline
\end{tabular}

\#: $95 \%$ confidence interval does not include 0 (significant at $p<0.05$ ).

was a significant drop in incidence in January, perhaps reflecting decreased transmission following the school holiday.

Aside from the seasonal variations in disease incidence, we also found that the risk of death from invasive pneumococcal pneumonia also varied seasonally among the elderly. These increases were especially pronounced during influenza epidemic periods, suggesting that influenza-associated cases might be more severe, but additional studies are needed to support this observation. This finding is consistent with clinical studies demonstrating that mixed influenza-bacterial infections have a higher case fatality than viral or bacterial infections alone [28]. The reason for the different seasonal pattern in case fatality between the elderly and the middle aged adults could be related to increased susceptibility to environmental stresses among the elderly.

These analyses focus on IPD (pneumococcal bacteraemia or meningitis), which constitutes a small percentage of all pneumococcal disease [29]. Studies of all-cause pneumonia suggest that influenza also accounts for a relatively small percentage of total cases [29]. More detailed analyses could focus on the effect of influenza on the incidence of all-cause pneumonia in different risk groups.

The ecological design of this study has both strengths and weaknesses. We have tried to control for unrelated seasonal factors by using harmonic terms, but we cannot draw a strong causal link due to the design. As a strength, ecological study designs do not suffer from biases in testing between age groups and are less affected by poor sensitivity of the viral tests.

We evaluated the relationship between influenza and pneumococcal disease with a 1-week lag. The impact of influenza on the risk for bacterial disease is thought to be transient, which is supported by the observation that pneumococcal disease cases quickly returned to baseline levels following the 2009 influenza pandemic [10].

There are some limitations of the dataset that we used. The "pneumonia" category was based on clinical judgment and subsequent ICD coding, so there is a potential for misclassification to occur. Such misclassification would probably reduce any differences between the pneumonia and nonpneumonia 
categories. Additionally, over the course of the 30-year study period, the rates and sensitivity of blood culturing and the reporting of isolates to SSI could have changed and could potentially influence the results reported here. Moreover, our definition of comorbidity is based on broad diagnoses covered by the Charlson index, and we might detect stronger differences if we could stratify by the type of comorbidity (e.g. immune-compromising conditions versus diabetes). Finally, we did not have information on the rate of blood culturing among hospitalised cases in the different age and comorbidity groups. If the rate of blood culturing during influenza periods differs between risk groups, this will introduce some confounding to the calculation of the estimates.

These analyses have demonstrated that the seasonal variations of pneumococcal disease incidence and severity differ between age groups, and the impact of influenza on pneumococcal disease differs by age, clinical presentation and comorbidity status. When comparing studies or projecting the impact of pneumococcal vaccine-associated changes, it will be necessary to take these heterogeneities into account.

\section{Acknowledgements}

The authors thank to K. Klugman (Bill and Melinda Gates Foundation, Seattle, WA, USA) for helpful discussions about these results. They would also like to thank T. Dalby, L. Pallesen and S. Hoffmann (Statens Serum Institut, Copenhagen, Denmark) for management and administrative support.

\section{References}

O'Brien KL, Wolfson LJ, Watt JP, et al. Burden of disease caused by Streptococcus pneumoniae in children younger than 5 years: global estimates. Lancet 2009; 374: 893-902.

2 Weinberger DM, Malley R, Lipsitch M. Serotype replacement in disease after pneumococcal vaccination. Lancet 2011; 378: 1962-1973.

3 Grabowska K, Högberg L, Penttinen P, et al. Occurrence of invasive pneumococcal disease and number of excess cases due to influenza. BMC Infect Dis 2006; 6: 58.

Hodges R, MacLeod C. Epidemic pneumococcal pneumonia. Am J Epidemiol 1946; 44: 231-236.

Klugman KP, Chien Y-W, Madhi SA. Pneumococcal pneumonia and influenza: a deadly combination. Vaccine 2009; 27: Suppl. 3, C9-C14.

6 Mahdi LK, Ogunniyi AD, LeMessurier KS, et al. Pneumococcal virulence gene expression and host cytokine profiles during pathogenesis of invasive disease. Infect Immun 2008; 76: 646-657.

7 McCullers JA. Insights into the interaction between influenza virus and pneumococcus. Clin Microbiol Rev 2006; 19: $571-582$.

8 Walter ND, Taylor TH, Shay DK, et al. Influenza circulation and the burden of invasive pneumococcal pneumonia during a non-pandemic period in the United States. Clin Infect Dis 2010; 50: 175-183.

9 Kuster SP, Tuite AR, Kwong JC, et al. Evaluation of coseasonality of influenza and invasive pneumococcal disease: results from prospective surveillance. PLoS Med 2011; 8: e1001042.

10 Weinberger DM, Simonsen L, Jordan R, et al. Impact of the 2009 influenza pandemic on pneumococcal pneumonia hospitalizations in the US. J Infect Dis 2012; 205: 458-465.

11 Madhi S, Petersen K, Madhi A, et al. Impact of human immunodeficiency virus type 1 on the disease spectrum of Streptococcus pneumoniae in South African children. Pediatr Infect Dis J 2000; 19: 1141-1147.

12 McCullers JA, McAuley JL, Browall S, et al. Influenza enhances susceptibility to natural acquisition of and disease due to Streptococcus pneumoniae in ferrets. J Infect Dis 2010; 202: 1287-1295.

13 Diavatopoulos DA, Short KR, Price JT, et al. Influenza A virus facilitates Streptococcus pneumoniae transmission and disease. FASEB J 2010; 24: 1789-1798.

14 Talbot T, Poehling KA, Hartert TV, et al. Seasonality of invasive pneumococcal disease: temporal relation to documented influenza and respiratory syncytial viral circulation. Am J Med 2005; 118: 285-291.

15 Techasaensiri B, Techasaensiri C, Mejías A, et al. Viral coinfections in children with invasive pneumococcal disease. Pediatr Infect Dis J 2010; 29: 519-523.

16 Edwards J, Markey PG, Cook HM, et al. The relationship between influenza and invasive pneumococcal disease in the Northern Territory, 2005-2009. Med J Aust 2011; 194: 207.

17 Weinberger DM, Simonsen L, Jordan R, et al. Impact of the 2009 influenza pandemic on pneumococcal pneumonia hospitalizations in the US. J Infect Dis 2012; 205: 458-465.

18 Blyth CC, Webb SA, Kok J, et al. The impact of bacterial and viral co-infection in severe influenza. Influenza Other Respir Viruses 2013; 7: 168-176.

19 Harboe ZB, Benfield TL, Valentiner-Branth P, et al. Temporal trends in invasive pneumococcal disease and pneumococcal serotypes over 7 decades. Clin Infect Dis 2010; 50: 329-337.

20 Harboe ZB, Thomsen RW, Riis A, et al. Pneumococcal serotypes and mortality following invasive pneumococcal disease: a population-based cohort study. PLoS Med 2009; 6: e1000081.

21 Zambon MC, Stockton JD, Clewley JP, et al. Contribution of influenza and respiratory syncytial virus to community cases of influenza-like illness: an observational study. Lancet 2001; 358: 1410-1416.

22 Nielsen J, Mazick A, Glismann S, et al. Excess mortality related to seasonal influenza and extreme temperatures in Denmark, 1994-2010. BMC Infect Dis 2011; 11: 350-350.

23 Mazick A, Christiansen AH, Samuelsson S, et al. Using sentinel surveillance to monitor effectiveness of influenza vaccine is feasible: a pilot study in Denmark. Euro Surveill 2006; 11: 254-256.

24 Politis D. Resampling time series with seasonal components. Frontiers in Data Mining and Bioinformatics: Proceedings of the 33rd Symposium on the Interface of Computing Science and Statistics, 2001. www. interfacesymposia.org/I01/master.pdf Date last accessed: October 10, 2013.

25 Serfling RE. Methods for current statistical analysis of excess pneumonia-influenza deaths. Pub Health Rep (18961970) 1963; 78: 494-506. 
Dowell S, Whitney CG, Wright C, et al. Seasonal patterns of invasive pneumococcal disease. Emerg Infect Dis 2003; 9: 573-579.

27 Walter ND, Taylor TH Jr, Dowell SF, et al. Holiday spikes in pneumococcal disease among older adults. $\mathrm{N}$ Engl J Med 2009; 361: 2584-2585.

28 von Baum H, Schweiger B, Welte T, et al. How deadly is seasonal influenza-associated pneumonia? The German Competence Network for Community-Acquired Pneumonia. Eur Respir J 2011; 37: 1151-1157.

29 Simonsen L, Taylor RJ, Young-Xu Y, et al. Impact of pneumococcal conjugate vaccination of infants on pneumonia and influenza hospitalization and mortality in all age groups in the United States. mBio 2011; 2: e00309-10. 